\title{
Stewart-Treves syndrome: a case report
}

\author{
DG McKeown, PJ Boland
}

\author{
Memorial Sloan-Kettering Cancer Center, New York, US
}

\section{ABSTRACT}

We present a case of chronic lymphoedema that progressed to Stewart-Treves syndrome in a 63-year-old woman with a previous modified radical mastectomy, associated lymph node dissection, chemotherapy and radiotherapy. While producing stabilisation of most cutaneous lesions initially, chemotherapeutic treatment of the angiosarcoma did not prevent subsequent metastasis and patient death. We urge vigilance and regular follow-up appointments for patients following a mastectomy with chronic lymphoedema to facilitate prevention or early treatment of this devastating syndrome.

\section{KEYWORDS}

Angiosarcoma - Stewart-Treves syndrome - Lymphoedema - Lymphangiosarcoma - Postmastectomy angiosarcoma - Breast cancer

Accepted 20 January 2013; published online 27 June 2013

\section{CORRESPONDENCE TO}

David McKeown, Memorial Sloan-Kettering Cancer Center, 1,275 York Avenue, Suite A342, New York, NY 10065, US T: +1 212639 8684; F: +1 212717 3573; E: mckeownd@mskcc.org

The eponymous Stewart-Treves syndrome refers to the development of an angiosarcoma from longstanding lymphoedema due to axillary lymph node dissection following a radical mastectomy. ${ }^{1}$ However, angiosarcoma can occur secondary to lymphoedema from other causes such as filariasis, radiotherapy or lymph node dissection. This case describes the diagnosis of an angiosarcoma in a patient presenting with the cutaneous manifestations of this disease.

\section{Case history}

A 63-year-old woman presented to our clinic with swelling of the right upper extremity. Her past medical history was significant for stage III adenocarcinoma of the right breast, treated 15 years previously with a modified radical mastectomy, axillary lymph node clearance and multiple cycles of chemotherapy with stem cell rescue. Postoperatively, she received 5,940cGy to the chest wall and 5,080cGy to the axilla. Shortly after her surgical and radiotherapy treatments, she developed progressive lymphoedema of the right upper limb.

The patient was in good health until 18 months ago, when she noticed bruising on her right upper limb. This transformed into nodularity, which spread to the anterior chest, including the site of her prior mastectomy. A biopsy of this skin lesion showed an angiosarcoma, which was positive for CD31, CD34 and factor VIII, all of which are consistent with Stewart-Treves syndrome.

The patient was seen initially by the medical oncology team and a chemotherapeutic regimen of doxorubicin was recommended, which stabilised most of the lesions. How- ever, a fungating lesion developed in the inner portion of the right limb, followed by the development of several other lesions along the posterior thorax. Computed tomography (Figs 1 and 2) demonstrated masses measuring $4.8 \mathrm{~cm} \mathrm{x}$ $2.1 \mathrm{~cm}$ in the anterior upper right arm and $5.2 \mathrm{~cm} \times 2.6 \mathrm{~cm}$ in the medial distal arm. This progression culminated in ulceration and massive blood loss 12 months later, secondary to the fungating lesions (Figs 3 and 4). She required 17 units of packed red blood cells and 2 units of platelets.

Palliative radiotherapy was considered although it was believed that such treatment might worsen her symptoms due to the need to treat the entire upper extremity. The patient was placed on palliative pain control and subsequently developed bilateral pulmonary emboli. Forequarter amputation of the affected limb was discussed but she did not wish to pursue this option. She was transferred to hospice care and succumbed to her illness shortly thereafter.

\section{Discussion}

Angiosarcoma may arise de novo as a primary tumour or it can develop secondary to chronic lymphoedema. The latter aetiology is characteristic of Stewart-Treves syndrome. This cutaneous angiosarcoma is exceedingly rare and usually fatal.

It was first described by Fred Stewart and Norman Treves in 1948 at Cornell Medical College in New York, where they reported six cases of angiosarcoma that arose from areas of lymphoedema as a consequence of mastectomy. ${ }^{1}$ The lesions tend to present as unexplained enlarging plaques of red/purple papules. They may occur anywhere in the 


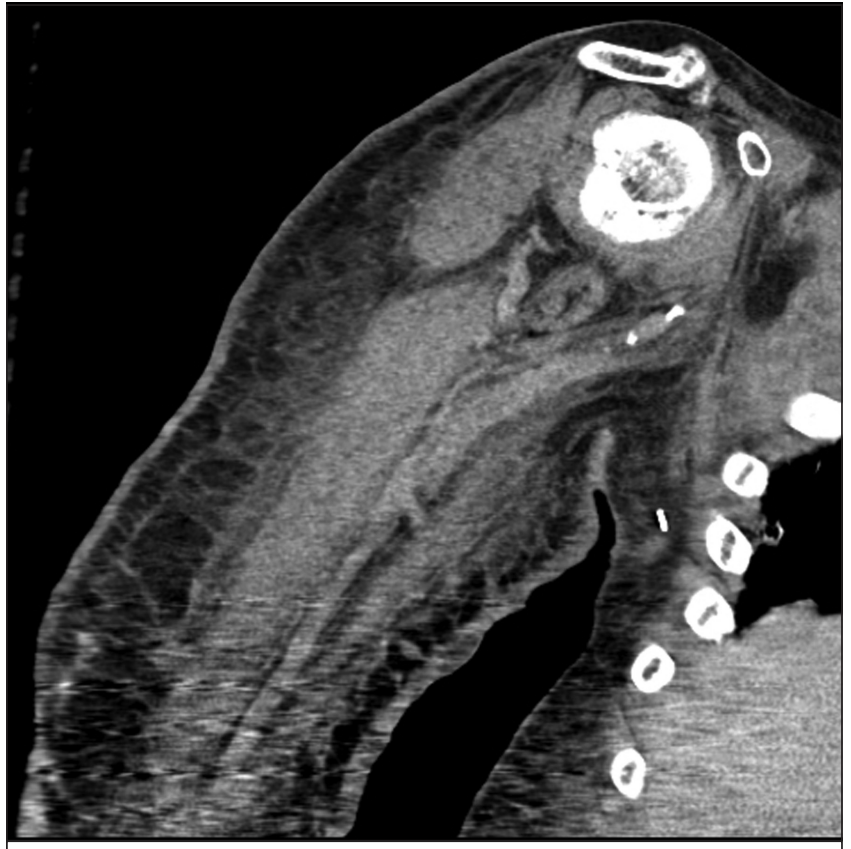

Figure 1 Coronal computed tomography of the right upper extremity demonstrating subcutaneous lymphoedema throughout the right upper limb

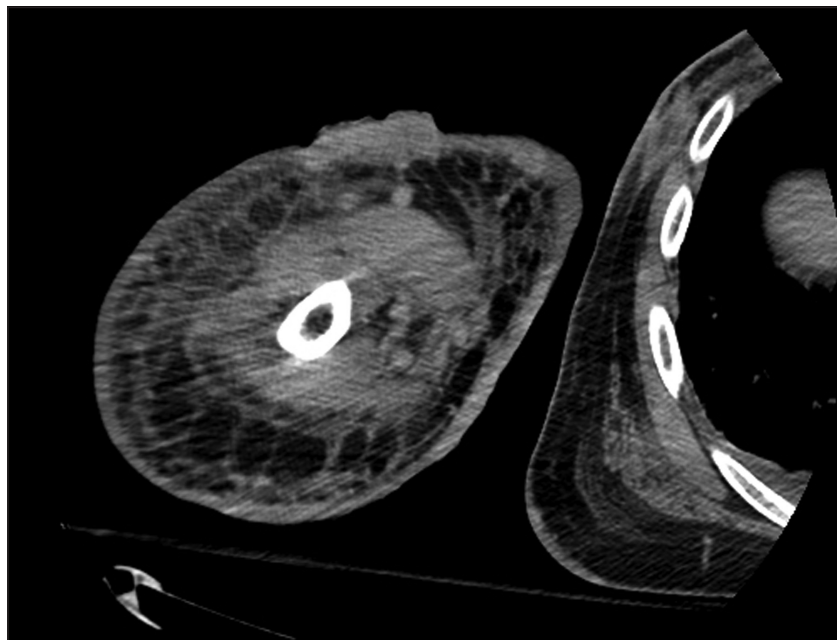

Figure 2 Axial computed tomography demonstrating a $4.8 \mathrm{~cm}$ $x 2.1 \mathrm{~cm}$ mass in the anterior upper right arm

affected limb as well as near or directly at the site of the previous mastectomy. Interestingly, Stewart and Treves noted that radiotherapy was not involved in the development of angiosarcoma in any of the cases reported in their series. Sarcomas induced following radiation occur typically in the previously radiated field, either located centrally or peripherally. However, angiosarcoma secondary to lymphoedema may occur anywhere in the affected limb or even at the

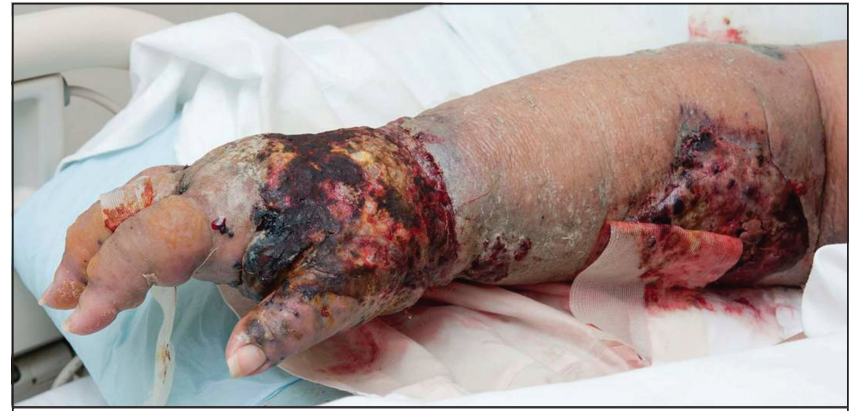

Figure 3 Fungating lesions on the dorsal aspect of the right hand and medial forearm

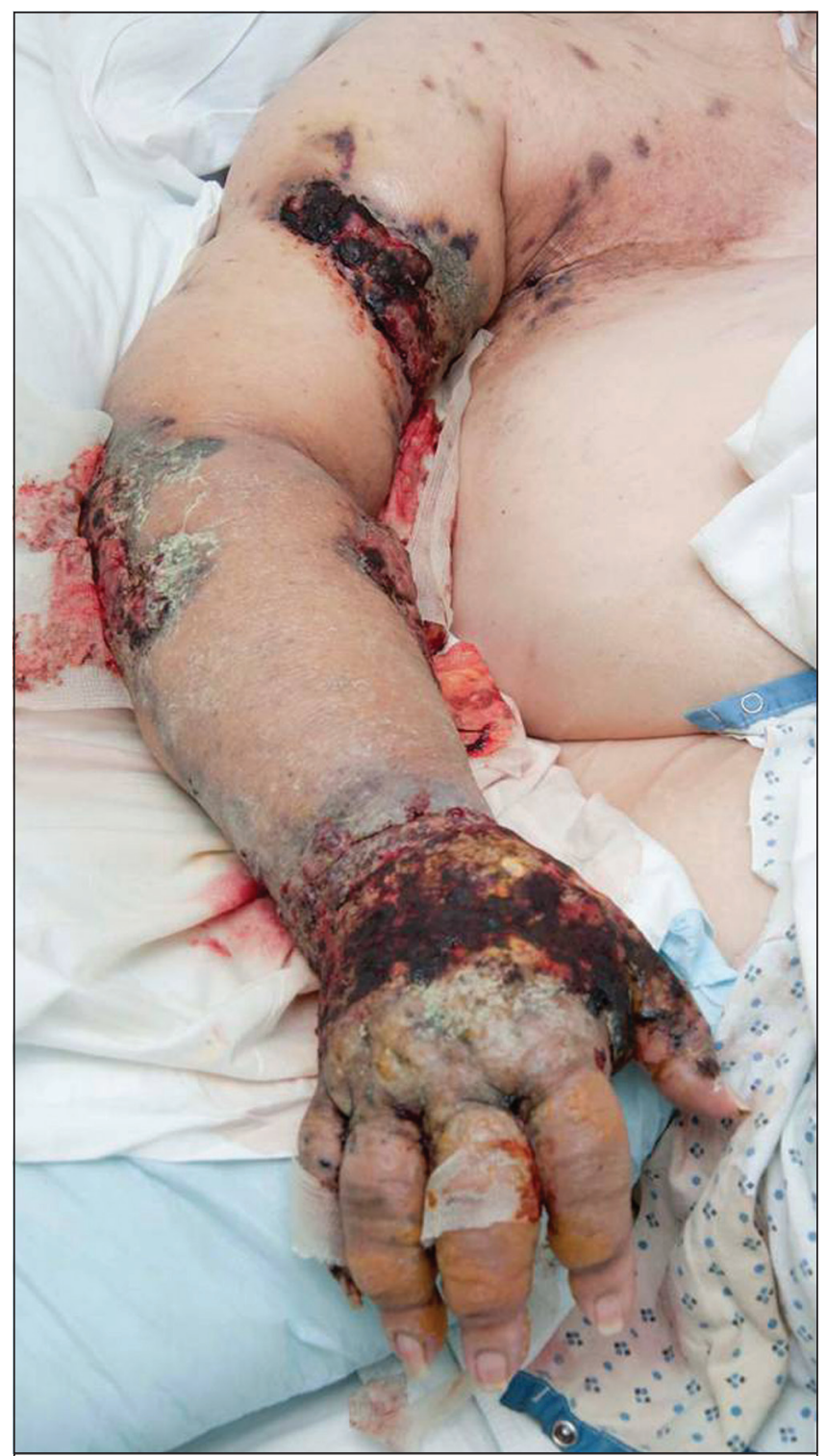

Figure 4 Fungating lesions on the dorsal aspect of the right hand, medial lateral aspect of the forearm and the inner portion of the right upper arm 
mastectomy site; it does not have to be present in the radiation field.

At present, there are over 400 cases of Stewart-Treves syndrome reported in the literature. The prevalence is estimated at approximately $0.45 \%$ in patients who survive longer than five years after a mastectomy. ${ }^{2}$ The incidence has been decreasing recently, most likely due to changes in operative therapy and radiotherapy in breast cancer patients. Conservative surgery to minimise loss of breast tissue, coupled with less axillary radiation treatment, has led to a reduction in chronic lymphoedema to as low as $4 \% .^{3}$ This reduction has also been aided by the advent of sentinel lymph node biopsy, a technique that has led to a decrease in unnecessary axillary lymph node clearance at the time of surgery.

The underlying pathophysiology of this disease is still debated. It is postulated that lymphatic blockage encourages the growth of lymphatic vessels through growth factors and cytokines. This is demonstrated by the fact that there is lymphatic vessel proliferation in unaffected areas of the affected limb. ${ }^{2}$ Radiotherapy is not directly linked with the development of angiosarcoma in the radiation field. Nevertheless, it may predispose to the development of lymphoedema secondary to axillary node sclerosis. Oedema associated with cardiac or renal disease is not known to be associated with the development of this syndrome.

Histologically, the diagnosis can be aided with positive staining for laminin, antibodies against factor VIII, type IV collagen and CD31. ${ }^{4}$ High level gene amplification of MYC is also a distinguishing feature of cutaneous secondary angiosarcomas, which develop after chronic lymphoedema or irradiation. This is in contrast to primary angiosarcomas, which tend to be present deep in soft tissue and do not have high MYC gene amplification. ${ }^{5}$

Treatment of this syndrome is difficult as angiosarcomas are all high grade, and typically metastasise to the lungs and chest wall. Patients who are treated with amputation rather than radiotherapy have a better prognosis. Despite this, their overall survival remains very poor with a survival range of 5-8 months. ${ }^{6}$ Prevention of this devastating disease may, in fact, prove to be the most effective treatment. Weight loss, compression bandaging, laser therapy, pressure devices and physiotherapy have all been proven to be effective in the treatment of lymphoedema.

\section{Conclusions}

This case demonstrates that despite the seemingly innocuous nature of chronic lymphoedema, it does have the potential to evolve into a much more devastating entity. We urge vigilance and regular follow-up appointments for patients following a mastectomy with chronic lymphoedema, particularly for those who have had surgery in the past 20-30 years prior to the introduction of sentinel lymph node biopsy and more accurate radiotherapy. Yearly surveillance coupled with preventative measures in these patients may prove vital in the prevention or early treatment of this devastating syndrome.

\section{References}

1. Stewart FW, Treves N. Lymphangiosarcoma in postmastectomy lymphedema; a report of six cases in elephantiasis chirurgica. Cancer 1948; 1: 64-81.

2. Lee SB, Cho BK, Houh W et al. A case of Stewart-Treves syndrome. J Korean Med Sci 1988; 3: 83-88.

3. Breidenbach M, Rein D, Schmidt T et al. Intra-arterial mitoxantrone and paclitaxel in a patient with Stewart-Treves syndrome: selection of chemotherapy by an ex vivo ATP-based chemosensitivity assay. Anticancer Drugs 2000; 11: 269-273.

4. Ordóñez NG, Batsakis JG. Comparison of Ulex europaeus I lectin and factor VIII-related antigen in vascular lesions. Arch Pathol Lab Med 1984; 108 129-132.

5. Manner J, Radlwimmer B, Hohenberger $\mathrm{P}$ et al. MYC high level gene amplification is a distinctive feature of angiosarcomas after irradiation or chronic lymphedema. Am J Pathol 2010; 176: 34-39.

6. Sharma A, Schwartz RA. Stewart-Treves syndrome: pathogenesis and management. J Am Acad Dermatol 2012; 67: 1,342-1,348. 